\title{
Cauda Equina Syndrome Caused by Spinal Dural Arteriovenous Fistula
}

\author{
Myung Jun Shin, M.D., Wan Kim, M.D., Seung Kug Baik, M.D. ', Soo Yeon Kim, M.D., Sung Nyun Kim, M.D.
}

Departments of Rehabilitation Medicine and ${ }^{1}$ Radiology, Pusan National University School of Medicine, Busan 602-739, Korea

Spinal dural arteriovenous fistula (SDAVF) is rare but still the most commonly encountered vascular malformation of the spinal cord. A 31-year-old male developed gait disturbance due to weakness of his lower extremities, voiding difficulty and sexual dysfunction with a progressive course since 3 months. He showed areflexia in both knees and ankles. Electromyographic findings were suggestive of multiple root lesions involving bilateral L2 to S4 roots of moderate degree. Magnetic resonance images showed high signal intensity with an ill-defined margin in T2-weighted images and intensely enhanced by a contrast agent through the lumbosacral spinal cord. Selective spinal angiography confirmed a dural arteriovenous fistula with a nidus at the L2 vertebral level. After selective endovascular embolization, his symptoms drastically improved except sexual dysfunction. We report a rare case of cauda equina syndrome due to spinal arteriovenous fistula with drastic improvement after endovascular embolization.

Key Words Spinal dural arteriovenous fistula, Cauda equina syndrome, Endovascular embolization

\section{INTRODUTION}

Spinal dural arteriovenous fistula (SDAVF) is a rare vascular malformation that can induce progressive upper motor neuron signs. Although it occasionally develops at the cervical region, more than $80 \%$ of these fistulas occur between $\mathrm{T} 6$ and $\mathrm{L} 2 .^{1-3} \mathrm{~A}$ recent study reported that men are affected 4-5 times more frequently than women

Received June 6, 2010; Accepted January 17, 2011

Corresponding author: Wan Kim

Department of Rehabilitation Medicine, Pusan National University Hospital, Pusan National University School of Medicine, 1-10, Ami-dong, Suh-gu, Busan 602-739, Korea

Tel: +82-51-240-7485, Fax: +82-51-247-7485, E-mail: wanykim920@gmail. com

(c) This is an open-access article distributed under the terms of the Creative Commons Attribution Non-Commercial License (http:// creativecommons.org/licenses/by-nc/3.0) which permits unrestricted noncommercial use, distribution, and reproduction in any medium, provided the original work is properly cited.

Copyright $\odot 2011$ by Korean Academy of Rehabilitation Medicine and that the mean age at the time of diagnosis is 55-60 years. ${ }^{2,3}$ Increased blood flow to the vein through a fistula causes venous congestion, which reduces blood supply to the spinal cord and nerve roots, induces neurological dysfunctions and subsequently manifests a variety of clinical symptoms. ${ }^{2,4}$ SDAVFs usually present with slowly progressive upper motor neuron signs such as paraplegia and voiding difficulty, and rarely present with pure lower motor neuron signs. ${ }^{3}$ These symptoms have been shown to gradually improve after surgical excision or endovascular embolization of the fistula, but there have been few cases of SDAVF that have shown rapid neurological recovery after treatment.

A 31-year-old male patient with progressive weakness of the lower extremities and voiding difficulty after a sudden onset of low back pain was diagnosed with cauda equina syndrome due to SDAVF and showed drastic improvement after endovascular embolization. We report 
this case with a brief review of the literature.

\section{CASE REPORT}

A 31-year-old male patient visited a regional hospital because he had experienced a 3-month history of sudden low back pain. The symptoms did not improve with oral medication, physical therapy, epidural steroid injection and electrical intramuscular stimulation at the L3-L4 level, and thereafter he had difficulty in getting up from chairs and putting on his pants. Moreover, voiding/ defecation difficulty, urinary/fecal incontinence and erectile dysfunction newly developed. The patient was eventually referred to our clinic.

There were neither a remarkable past nor family histories found upon evaluation. At presentation, the visual analogue scores (VAS) for low back pain and right leg pain were 8 points each. On physical examination, a $3 / 5$ grade of motor function was noted in the hip flexors, extensors and abductors, and the knee flexors on both sides, as well as a $4 / 5$ grade of motor function in the knee extensors and the ankle dorsiflexors and plantar flexors on both sides. There was also hyperesthesia accompanied by allodynia on the lateral surface of the lower extremities and foot dorsum on both sides, as well as paresthesia including numbness, on both of his fifth toes. Neither deep tendon reflexes nor pathologic reflexes were evoked in the lower extremities. Motor and sensory functions in the sacral segment had decreased. Anal reflex and bulbocavernosus reflex were not elicited.

The results of routine hematologic tests and nerve conduction studies were normal. Needle electromyography of the muscles innervated by L2 to S1 segments revealed (1) increased insertional activities, (2) moderate abnormal resting potentials with positive sharp waves and fibrillation potentials, and (3) decreased recruitment of motor units with long duration and increased polyphasic potentials. Based on these findings,
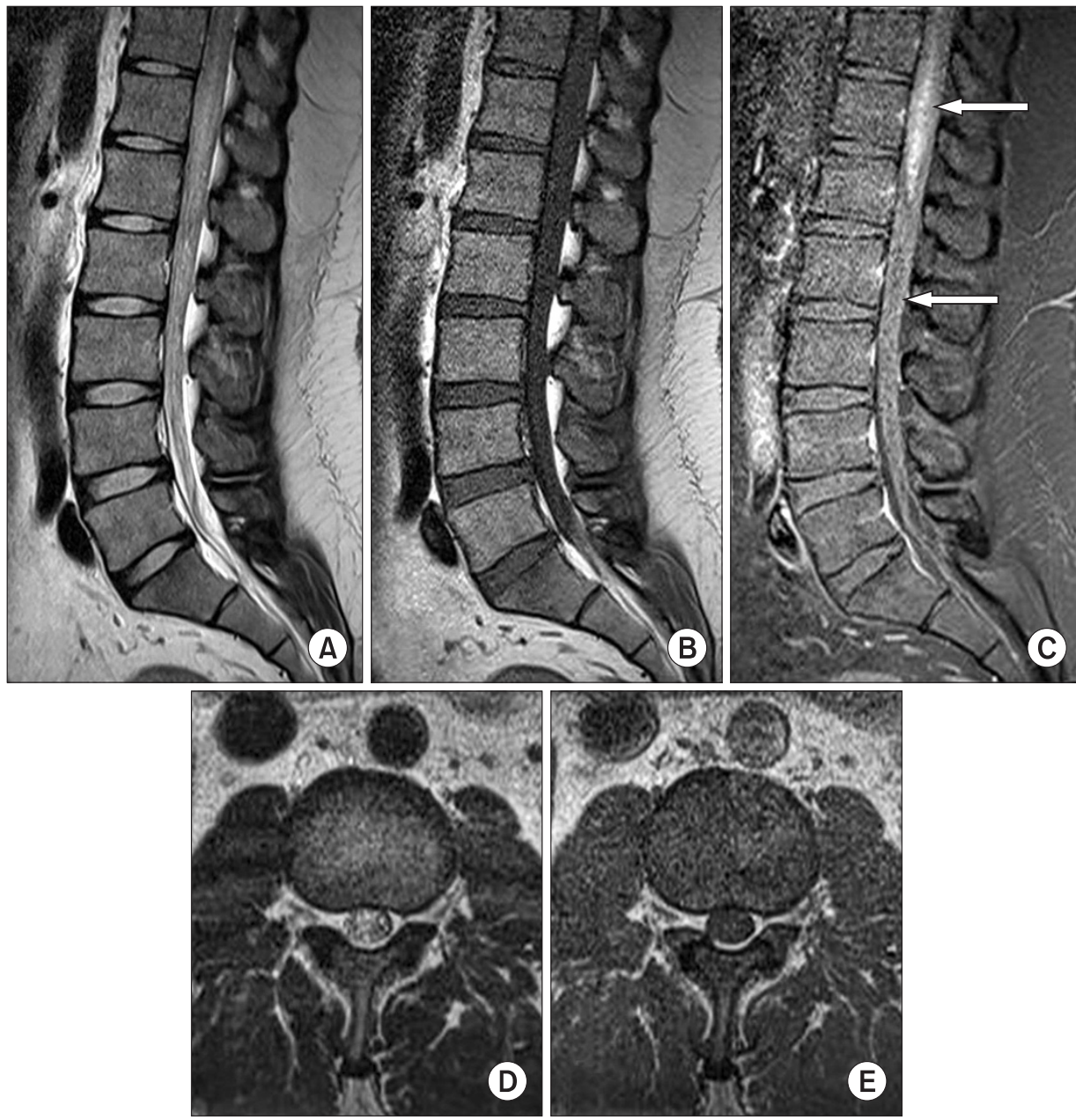

Fig. 1. T2-weighted magnetic resonance (MR) images $(\mathrm{A}, \mathrm{D})$ show central intramedullary hyperintensity of the distal cord and cauda equina as a sign of central myelopathy with extensive spinal cord edema. T1-weighted (B, E), pregadolinium and (C) postgadolinium MR images demonstrate intramedullary hyperintensity with flow voids (white arrow) on the dorsal aspect of the spinal cord corresponding to the dilated perimedullary veins. 
the patient was diagnosed with cauda equina syndrome.

Magnetic resonance (MR) imaging of the lumbar spines revealed edema of the distal spinal cord and cauda equina along with increased intensity and a scattered signal void of dilated veins on T2-weighted images, which was suggestive of SDAVF (Fig. 1). Spinal angiography exhibited SDAVF at the L2-L3 level and a small atypical cyst adjacent to the fistula (Fig. 2). Selective endovascular embolization was performed with a mixture of $33 \%$ n-butyl cyanoacrylate and lipiodol. Angiography taken after the procedure revealed a complete occlusion of the fistula (Fig. 2). Two days after the procedure, pain in the lumbar area and lower extremities was decreased to 2 points on the VAS, and voiding difficulty was improved. Three days after the procedure, physical examination showed a $4 / 5$ grade of motor function in the hip flexors, extensors and abductors and the knee flexors on both sides, as well as a 5/5 grade in the knee extensors and ankle dorsiflexors and plantar flexors on both sides. Seven days after the procedure, physical examination showed that motor function was recovered to normal grade except a 4/5 grade in the knee flexors. The patient was able to ambulate without any walking aids and his sensory function was normal. Deep tendon reflexes except ankle jerk on the right side were normal. Symptoms, including defecation difficulty, voiding difficulty, urinary incontinence and fecal incontinence, were remarkably improved. However, sexual dysfunctions such as erectile difficulty remained unchanged.

Electromyography taken 2 weeks after the procedure revealed an increase in the amplitude of motor evoked potentials but no change in its latency. In addition, it revealed that there were no significant changes in amplitude and latency of somatosensory evoked potentials (Table 1). Needle electromyography of the muscles innervated by L2 to S1 segments revealed that insertional activities increased but interference patterns improved.

MR imaging performed 2 weeks after the procedure exhibited decreased signal intensity in the distal spinal


Fig. 2. Spine anteroposterior angiogram with selective catheterization of the left L2 segmental artery (white arrow) shows a fistula draining to the perimedullary vein (black arrow) (A, B). A small sac (asterisk) is noted near the fistula which is not observed in typical dural arteriovenous fistulas (B). After embolization using a NBCA/lipiodol mixture, no further residual perfusion of the fistula was demonstrated (C, D). 
Table 1. Changes in Electrophysiologic Findings Following Endovascular Embolization

\begin{tabular}{|c|c|c|c|}
\hline \multirow{2}{*}{$\overline{\text { SEPs }}$} & \multirow{2}{*}{$\frac{\text { At diagnosis }}{\text { ior tibial nerve }}$} & \multicolumn{2}{|c|}{ At follow-up } \\
\hline & & & \\
\hline & \multirow[t]{2}{*}{ Latency (P37/N45, msec) } & R $42.9 / 51.1$ & R $42.8 / 51.2$ \\
\hline & & L $42.2 / 50.4$ & L $42.7 / 51.4$ \\
\hline \multirow{2}{*}{\multicolumn{2}{|c|}{ Amplitude $(\mu \mathrm{V})$}} & R 0.55 & R 1.03 \\
\hline & & L 0.73 & L 0.88 \\
\hline \multicolumn{4}{|c|}{ Pudendal nerve } \\
\hline & Latency (msec) & 45.0 & 46.4 \\
\hline & Amplitude $(\mu \mathrm{V})$ & 1.01 & 0.35 \\
\hline \multicolumn{4}{|c|}{ MEPs Tibialis anterior muscle } \\
\hline \multirow{2}{*}{\multicolumn{2}{|c|}{ Latency $(\mathrm{msec})$}} & R 33.0 & R 32.4 \\
\hline & & L 32.6 & L 33.1 \\
\hline \multirow{2}{*}{\multicolumn{2}{|c|}{ Amplitude (mV) }} & R 0.54 & R 6.2 \\
\hline & & L 0.71 & L 6.2 \\
\hline \multicolumn{2}{|c|}{ BCRL Bulbocavernosus muscle } & $\begin{array}{c}\text { Not } \\
\text { obtainable }\end{array}$ & $\begin{array}{c}\text { Not } \\
\text { obtainable }\end{array}$ \\
\hline
\end{tabular}

SEPs: Somatosensory evoked potentials, MEPs: Motor evoked potentials, BCRL: Bulbocavernosus reflex latency, R: Right, L: Left

cord and the dura of the lumbar segment, resolution in spinal cord edema, remarkable reduction in the thickness of the spinal cord and decreased signal void compared to those before the procedure (Fig. 3).

\section{DISCUSSION}

In 1926, Foix and Alajouanine ${ }^{5}$ first reported subacute necrotic myelitis as SDAVF in the autopsy of a patient with slowly progressive paraplegia and sensory dysfunction. In patients with SDAVF, neurological symptoms progress slowly due to dysfunctions of the spinal cord and nerve roots caused by venous congestion. SDAVFs should be differentiated from other spinal diseases in patients with low back pain or paraplegia because it can be improved through accurate diagnosis and adequate treatment. However, since SDAVFs are manifested by nonspecific symptoms and their prevalence is extremely rare, they are easy to overlook when clinicians diagnose patients with low back pain or motor dysfunction that are frequently seen at the department of rehabilitation medicine.

The mean onset age of SDAVFs is reported to be between 55 and 60 years $^{2,3}$ and the median age at the onset of initial symptoms is reported to be 57.6 years. ${ }^{3}$ The onset age of
SDAVF in our patient was as early as 31 years. Although he was clinically diagnosed with intervertebral disc degeneration and received conservative treatment, his symptoms were aggravated. Additional comprehensive studies established a confirmative diagnosis of SDAVF at our clinic.

The exact mechanism underlying the origin of SDAVFs is unknown, although they are considered to be acquired vascular malformations, probably as a result of infection, syringomyelia, trauma and operation. ${ }^{4}$ Arterial blood drains into the spinal veins through the SDAVF, which increases venous pressure and decreases the arteriovenous pressure difference. Then, decreased drainage into the spinal veins induces venous congestion accompanied by cord edema and develops chronic hypoxia and progressive myelopathy. ${ }^{2,4}$

Jellema et al. ${ }^{3}$ have reported that the most frequent initial symptom is gait disturbance (34\%), followed by numbness (24\%) and low back pain (14\%). They have also reported that clinical symptoms and signs at the time of diagnosis are micturition problems (80\%), leg weakness (78\%) and numbness (69\%). Our patient's initial symptom was low back pain, and following that he exhibited these additional symptoms as well. In typical cases of SDAVFs, upper motor neuron signs slowly progress and worsen over a period of $15-23$ months. ${ }^{3,4,6,7}$ It is interesting to note that in our case, cauda equina syndrome with pure lower motor neuron signs and no upper motor neuron signs developed over a relatively short period of 3 months.

Myelography was used to diagnose SDAVFs in the past, but MR imaging is currently used. ${ }^{4}$ In particular, digital subtraction angiography of the spinal cord enables clinicians to identify the location of the SDAVF, as well as feeding arteries, collateral feeders and venous drainage and to perform selective endovascular embolization. ${ }^{2,4}$ In our case, MR imaging revealed the typical findings of SDAVFs, while spinal angiography showed a small sac adjacent to the fistula. This is an intriguing finding in that cystic lesions rarely occur in SDAVFs, low-flow shunts. ${ }^{2,8}$ Hassler et al. ${ }^{9}$ have reported, in a local hemodynamics of SDAVFs by microvascular doppler ultrasonography during operation, that the mean venous pressure in SDAVFs is approximately $74 \%$ of the systemic arterial pressure. The occurrence of the venous sac is probably due to this high venous pressure.

The treatment modalities for SDAVFs include 



Fig. 3. T2-weighted (A, D), T1weighted $(B, E)$, pregadolinium and (C) postgadolinium spinal MR images performed 2 weeks after endovascular embolization. Compared to previous MR images (Fig. 1), they show reduced enhancement and edema of the distal cord with diminished flow voids.

endovascular embolization and open spinal surgery that disconnect an intradural shunting vein from the perimedullary venous plexus in order to eliminate venous congestion of the spinal cord before irreversible neurological damage. ${ }^{2,4,6,8}$ The current trend is that the first therapeutic approach should be endovascular, using a liquid embolic agent after fistulas and their feeders are assessed by angiography., ${ }^{2,10}$ Our patient was diagnosed with SDAVF by spinal angiography and underwent immediate selective endovascular embolization with liquid embolic agents.

The functional outcome of SDAVFs is related to various factors including the duration of symptoms, the degree of disability before treatment and success of fistula closure with the initial treatment. ${ }^{2,8}$ The prognosis of SDAVFs is generally better in younger patients with milder symptoms or a shorter interval between onset and treatment. ${ }^{2,8,10}$ It has been demonstrated that voiding difficulty, defecation difficulty, erectile dysfunction, pain and muscle spasm are symptoms relatively resistant to recovery. ${ }^{2,8}$ In our case, after endovascular embolization, symptoms including motor weakness, sensory dysfunction, voiding difficulty and pain, were rapidly improved but erectile dysfunction still persisted. This rapid improvement in clinical course was different from those of slow symptomatic improvement in previously reported cases. ${ }^{6,7,10}$ A plausible explanation for this may be that our patient was relatively young, diagnosed as early as 3 months after symptom onset, and received endovascular treatment immediately thereafter.

To conclude, here we have reported the case of a 30-year-old male patient with SDAVF who complained of low back pain and weakness in his lower extremities and voiding difficulty, who showed a rapid recovery after 
endovascular embolization. Clinicians should remember that SDAVFs can cause cauda equina syndrome with pure lower motor neuron signs and no upper motor neuron signs. Therefore, early diagnosis and treatment are mandatory to prevent irreversible neurological deficits because a delay in diagnosis and treatment may aggravate clinical course.

\section{REFERENCES}

1. Kendall BE, Logue V. Spinal epidural angiomatous malformations draining into intrathecal veins. Neuroradiology 1977; 13: 181-189

2. Krings T, Geibprasert S. Spinal dural arteriovenous fistulas. Am J Neuroradiol 2009; 30: 639-648

3. Jellema K, Canta LR, Tijssen CC, van Rooij WJ, Koudstaal PJ, van Gijn J. Spinal dural arteriovenous fistulas: clinical features in 80 patients. J Neurol Neurosurg Psychiatry 2003; 74: 1438-1440

4. Koch C. Spinal dural arteriovenous fistula. Curr Opin Neurol 2006; 19: 69-75

5. Foix C, Alajouanine T. Subacute necrotic myelitis, slowly progressive central myelitis with vacular hyperplasia, and slowly ascending, increasingly flaccid amyotrophic paraplegia accompanied by albuminocytologic dissociation. Rev Neurol 1926; 33: $1-42$

6. Schick U, Hassler W. Treatment and outcome of spinal dural arteriovenous fistulas. Eur Spine J 2003; 12: 350355

7. Park SB, Han MH, Jahng TA, Kwon BJ, Chung CK. Spinal dural arteriovenous fistulas: clinical experience with endovascular treatment as a primary therapeutic modality. J Korean Neurosurg Soc 2008; 44: 364-369

8. Prieto R, Pascual JM, Gutierrez R, Santos E. Recovery from paraplegia after the treatment of spinal dural arteriovenous fistula: case report and review of the literature. Acta Neurochir 2009; 151: 1385-1397

9. Hassler W, Thorn A, Grote EH. Hemodynamics of spinal dural arteriovenous fistulas. An intraoperative study. J Neruosurg 1989; 70: 360-370

10. Andres RH, Barth A, Guzman R, Remonda L, ElKoussy M, Seiler RW, Widmer HR, Schroth G. Endovascular and surgical treatment of spinal dural arteriovenous fistulas. Neruoradiology 2008; 50: 869876 